\title{
A THEOREM ON BIQUADRATIC RECIPROCITY
}

\author{
EZRA BROWN
}

ABSTRACT. The following theorem on biquadratic reciprocity is proved: if $p \equiv q \equiv 1(\bmod 4)$ are primes for which $(p \mid q)=1$, and if $p=r^{2}+q s^{2}$ for some integers $r$ and $s$, then

$$
\begin{aligned}
(p \mid q)_{4}(q \mid p)_{4} & =1, & & \text { if } q \equiv 1(\bmod 8) ; \\
& =(-1)^{2}, & & \text { if } q \equiv 5(\bmod 8) .
\end{aligned}
$$

Simple expressions for the biquadratic character of some small primes are also obtained.

$\mathrm{K}$. Burde [2] has proven the following interesting theorem about biquadratic reciprocity:

ThEOREM 1. If $p=a^{2}+b^{2}, q=c^{2}+d^{2}, a \equiv c \equiv 1, b \equiv d \equiv 0(\bmod 2)$, $a b>0, c d>0, p$ and $q$ are primes, and $(p \mid q)=1$, then $(p \mid q)_{4}(q \mid p)_{4}$ $=(-1)^{(p-1) / 4}(a d-b c \mid p)$.

If it happens that $p$ can be written as $r^{2}+q s^{2}$, with $r$ and $s$ integers, then Burde's results acquire a particularly simple form. We shall prove the following theorem:

THEOREM 2. If $p \equiv q \equiv 1(\bmod 4)$ are primes such that $(p \mid q)=1$, and $p$ is representable as $r^{2}+q s^{2}$, where $r$ and s are integers, then

$$
\begin{aligned}
(p \mid q)_{4}(q \mid p)_{4} & =1, & & q \equiv 1(\bmod 8) ; \\
& =(-1)^{8}, & & q \equiv 5(\bmod 8) .
\end{aligned}
$$

Throughout this paper, we assume that $p$ and $q$ satisfy the hypotheses in Theorem $1 ;(p \mid q)$ is the Legendre symbol, and we write $(p \mid q)_{4}=1$ or -1 according as $p$ is or is not a biquadratic residue $(\bmod q)$.

LEMмA 1. All prime solutions of the diophantine equation

$$
a^{2}+b^{2}=r^{2}+q s^{2}
$$

are contained in the following sets of expressions:

Received by the editors January 19, 1971.

AMS 1970 subject classifications. Primary 10A15; Secondary 10B05, 10C05.

Key words and phrases. Power residues, biquadratic residues, reciprocity, quadratic diophantine equations. 
(2)

$$
\begin{aligned}
& a=c\left(t_{0}^{2}+t_{1}^{2}-t_{2}^{2}-t_{3}^{2}\right)+2 d\left(-t_{0} t_{2}+t_{1} t_{3}\right) \\
& b=2 c\left(-t_{0} t_{3}+t_{1} t_{2}\right)+2 d\left(t_{0} t_{1}+t_{2} t_{3}\right) \\
& \left.r=c\left(t_{0}^{2}+t_{3}^{2}-t_{1}^{2}-t_{2}^{2}\right)+2 d\left(-t_{0} t_{2}-t_{1} t_{3}\right) \quad \text { (if } s \text { is even }\right) \\
& s=2\left(-t_{0} t_{1}+t_{2} t_{3}\right), \\
& a=c\left(t_{0}^{2}+t_{1}^{2}-t_{2}^{2}-t_{3}^{2}\right)+2 d\left(t_{0} t_{3}+t_{1} t_{2}\right) \\
& \left.b=2 c\left(-t_{0} t_{3}+t_{1} t_{2}\right)+d\left(t_{0}^{2}+t_{2}^{2}-t_{1}^{2}-t_{3}^{2}\right) \quad \text { (if } s \text { is odd }\right) . \\
& r=2 c\left(t_{0} t_{1}-t_{2} t_{3}\right)+2 d\left(t_{0} t_{2}+t_{1} t_{3}\right) \\
& s=t_{0}^{2}+t_{3}^{2}-t_{1}^{2}-t_{2}^{2}
\end{aligned}
$$

Here, the $t_{i}$ are independent integer-valued parameters, one or three of which are odd.

Proof. See $[1, \S 5]$. The roles of $x_{1}, x_{2}, x_{5}$ and $x_{6}$ in [1] are taken here by $a, b, r$ and $s$, respectively.

LEMMA 2. For any prime solution of (1), we have $(c b-d a \mid q)=(2 \mid q)$ if $s$ is even, and $(c b-d a \mid q)=1$ if $s$ is odd.

Proof. If $s$ is even, then from (2) we have

$$
\begin{aligned}
c b-d a= & 2 c^{2}\left(-t_{0} t_{3}+t_{1} t_{2}\right)+2 c d\left(t_{0} t_{1}+t_{2} t_{3}\right) \\
& -c d\left(t_{0}^{2}+t_{1}^{2}-t_{2}^{2}-t_{3}^{2}\right)-2 d^{2}\left(-t_{0} t_{2}+t_{1} t_{3}\right) \\
\equiv & -c d\left(\left(t_{1}-t_{0}\right)^{2}-\left(t_{2}+t_{3}\right)^{2}\right)-2 d^{2}\left(t_{1}-t_{0}\right)\left(t_{2}+t_{3}\right)(\bmod q),
\end{aligned}
$$

since $c^{2} \equiv-d^{2}(\bmod q)$. Multiplying both sides by $d$, we obtain the congruence

$$
d(c b-d a) \equiv-c\left(c\left(t_{1}-t_{0}\right)+d\left(t_{2}+t_{3}\right)\right)^{2}(\bmod q) .
$$

Now

$$
\begin{aligned}
(c b-d a)(c b+d a) & =c^{2}\left(a^{2}+b^{2}\right)-a^{2}\left(c^{2}+d^{2}\right) \\
& =c^{2} p-a^{2} q \equiv c^{2} p \not \equiv 0(\bmod q),
\end{aligned}
$$

since $(c, q)=1$. Hence $c b-d a \neq 0(\bmod q)$ and we may write $(c b-d a \mid q)$ $=(-1 \mid q)(c \mid q)(d \mid q)$. But by Theorem 5 of $[1],(c \mid q)=1$ and $(d \mid q)$ $=(2 \mid q)$; hence $(c b-d a \mid q)=(2 \mid q)$, since $q \equiv 1(\bmod 4)$. Proof of the second statement is similar, relying on the expressions in (3), and is omitted.

Proof of Theorem 2. By Lemma 2 and Theorem 1 , if $s$ is even, then (reversing the roles of $p$ and $q$ in Theorem 1) $(p \mid q)_{4}(q \mid p)_{4}$ 
$=(-1)^{(q-1) / 4}(2 \mid p)=1$, since $q \equiv 1(\bmod 4)$. If $s$ is odd, then $(q \mid p)$, $(q \mid p)_{4}=(-1)^{(q-1) / 4}=1$ or -1 according as $q \equiv 1$ or $5(\bmod 8)$. Hence $(p \mid q)_{4}(q \mid p)_{4}=1$ or $(-1)^{8}$, according as $q \equiv 1$ or $5(\bmod 8)$.

As an application we determine the biquadratic characters of some small primes. Let $q=5$ or 13 and let $p \equiv 1(\bmod 4)$ be a prime such that $(p \mid q)=1$. It can be shown, using Thue's lemma on linear congruences, that every such prime is representable as $r^{2}+q s^{2}$, with integral $r$ and $s$. It is also the case that $(p \mid q)_{4}=1$ or -1 according as $[(p-1) / q]$ is even or odd (here $[X]=$ greatest integer in $X)$. For, $(p \mid 5)_{4}=1$ or -1 according as $p \equiv 1$ or $9(\bmod 10),(p \mid 13)_{4}=1$ if $p \equiv 1$, 3 or $9(\bmod 26)$, and $(p \mid 13)_{4}=-1$ if $p \equiv 17,23$ or $25(\bmod 26)$. This information, together with Theorem 2, yields the following result.

TheOREM 3. Let p be a prime $\equiv 1(\bmod 4)$. Then:

(a) If $(p \mid 5)=1$, then $(5 \mid p)_{4}=(-1)^{s+[(p-1) / 5]}$, where $p=r^{2}+5 s^{2}$.

(b) If $(p \mid 13)=1$, then $(13 \mid p)_{4}=(-1)^{s+[(p-1) / 13]}$, where $p=r^{2}+13 s^{2}$.

It also happens that every prime $p \equiv 1(\bmod 4)$ such that $(p \mid 37)=1$ can be represented as $r^{2}+37 s^{2}$. The conditions that $(p \mid 37)_{4}$ be 1 or -1 are not particularly simple: $(p \mid 37)_{4}=1$ or -1 according as $\left[\left(p^{9}-1\right) / 37\right]$ is odd or even. Nevertheless, we do have the following.

THEOREM 4. If $p \equiv 1(\bmod 4)$ is a prime such that $(p \mid 37)=1$, then $(37 \mid p)_{4}=(-1)^{s}(p \mid 37)_{4}$, where $p=r^{2}+37 s^{2}$.

\section{REFERENCES}

1. Ezra Brown, Representations of discriminantal divisors by binary quadratic forms, J. Number Theory 3 (1971).

2. Klaus Burde, Ein rationales biquadratisches Reziprozitätsgesetz, J. Reine Angew. Math. 235 (1969), 175-184. MR 39 \#2694.

Virginia Polytechnic Institute and State University, Blacksburg, VirGINIA 24061 